GRASAS Y ACEITES 71 (2)

April-June 2020, e353

ISSN-L: 0017-3495

https://doi.org/10.3989/gya.0117191

\title{
Exploring harmony in extra virgin olive oils and vegetables pairings
}

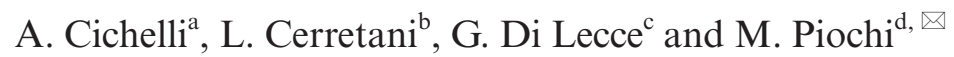 \\ a'Department of Medical, Oral and Biotechnological Science, University "G. d'Annunzio" of Chieti-Pescara, \\ Via dei Vestini, 66100, Chieti Scalo, $(\mathrm{CH})$, Italy \\ ${ }^{\mathrm{b}}$ S.A.L.P.A. Roseto, via nazionale 544, 64026, Roseto degli Abruzzi, Teramo (TE), Italy \\ 'Oleificio Zucchi S.p.A., via Acquaviva 12, 26100, Cremona (CR), Italy \\ ${ }^{\mathrm{d}}$ University of Gastronomic Sciences, Piazza Vittorio Emanuele 9, 12060, Bra (CN), Italy \\ Corresponding author: m.piochi@unisg.it
}

Submitted: 28 January 2019; Accepted: 17 April 2019; Published online: 17 June 2020

SUMMARY: Despite the growing interest in the sensory and healthy properties of extra virgin olive oil (EVOO), the topic of oil-food pairing is practically unexplored. This study systematically explores sensory effects on the flavor and harmony obtained by combining two ingredients (EVOOs and vegetables) and aims to provide practical indications for harmonic oil-vegetable combinations. The approach considered an optimal pairing of five EVOOs combined with five typical raw Italian vegetables characterized by different degrees of bitterness (Artichoke, Late Treviso radicchio, Chioggia radicchio, rocket, Early Treviso radicchio). An Index of Disharmony was computed for each pairing, using intensity ratings given by a trained panel that described EVOOs, vegetables and pairings. The results suggested a flavor congruency principle to enhance the oil-pairing harmony. EVOOs with intense green and bitter flavor maximise harmony when combined with very bitter or very pungent vegetables. EVOOs with moderate green flavor and bitterness seemed best paired with vegetables with low/intermediate bitterness.

KEYWORDS: Bitter; Extra virgin olive oil; Food pairing; Green fruity; Radicchio; Vegetables

RESUMEN: Explorando la armonía en los aceites de oliva virgen extra y maridajes con vegetales. A pesar del creciente interés por las propiedades sensoriales y saludables del aceite de oliva virgen extra (AOVE), el tema de la combinación de aceite y alimentos está casi inexplorado. Este estudio explora sistemáticamente los efectos sensoriales sobre el sabor y la armonía que se obtienen al combinar dos ingredientes (AOVE y vegetales) y tiene como objetivo proporcionar indicaciones prácticas para las combinaciones de aceites armónicos y vegetales. El enfoque consideró el emparejamiento óptimo de cinco EVOO combinados con cinco vegetales crudos italianos típicos caracterizados por diferentes grados de amargor (alcachofa, Late Treviso radicchio, Chioggia radicchio, Rúcula, Early Treviso radicchio). Se calculó un índice de desarmonía para cada emparejamiento, utilizando las calificaciones de intensidades dadas por un panel capacitado que describía los AOVE, las verduras y los emparejamientos. Los resultados sugirieron un principio de congruencia del sabor para mejorar la armonía de emparejamiento de aceites. Los AOVE con intenso sabor verde y amargo maximizan la armonía cuando se combinan con vegetales muy amargos o muy picantes. Los AOVE que tienen un sabor verde y un amargor moderados parecían combinarse mejor con los vegetales con un amargor bajo / intermedio.

PALABRAS CLAVE: Aceite de oliva virgen extra; Afrutado verde; Amargo; Maridaje de alimentos; Radicchio; Vegetales

ORCID ID: Cichelli A https://orcid.org/0000-0002-2987-5738, Cerretani L https://orcid.org/0000-0003-2372-5576, Di Lecce G https://orcid.org/0000-0001-7620-418X, Piochi M https://orcid.org/0000-0002-5934-1171

Citation/Cómo citar este artículo: Cichelli A, Cerretani L, Di Lecce G, Piochi M. 2020. Exploring harmony in extra virgin olive oils and vegetables pairings. Grasas Aceites 71 (2), e353. https://doi.org/10.3989/gya.0117191

Copyright: (C2020 CSIC. This is an open-access article distributed under the terms of the Creative Commons Attribution 4.0 International (CC BY 4.0) License. 


\section{INTRODUCTION}

Extra virgin olive oils (EVOOs) are defined by the European Community as those "oils obtained from the fruit of the olive tree solely by mechanical or other physical means under conditions that do not lead to alterations in the oil" (EEC Reg. 2568/91 and following modifications). EVOO flavor and culinary uses are among those factors responsible for the success of this type of oil among consumers with different origins. However, some peculiar sensory properties of EVOO, such as bitter taste or pungency, may be perceived as a barrier by consumers, especially those less familiar with EVOO (Recchia et al., 2012). Typically, the bitter taste and the pungency in oil may represent negative sensory attributes for consumers' liking (Caporale, Policastro and Monteleone, 2004). The bitterness in virgin olive oil is due to the presence of phenolic compounds, mainly aglycones and other secoiridoid derivatives, e.g., aglycones of oleuropein and ligstroside with antioxidant effects (Gutiérrez-Rosales et al., 2003). Bitterness in vegetables is induced by different types of chemical compounds, such as glucosinolates and isothiocyanates (Drewnowski and Gomez-Carneros, 2000), and pungency is due to the presence of glucosinolates (Pasini et al., 2011). Vegetables are well recognised for being beneficial to human health, thanks to their protective function against cancer and other chronic degenerative diseases (Van Duyn and Pivonka, 2000). Unfortunately, as found for EVOOs, vegetable bitterness has also been suggested to cause rejection among consumers, especially children (Keller et al., 2002). In order to valorize EVOO as a product, it is important to study the effect of EVOO in combination with different food matrices in order to propose appropriate commercial strategies that take into account consumer perceptions. Few studies documented the effect of combining EVOOs with different food matrices, and particularly with raw vegetables, while non-academic professionals (e.g. chef and gourmet bloggers) often address the issue of food pairing (oil combined with food). The few systematic studies existing on food pairing tend to focus on the combination of two different products, typically a food and a beverage, which can also be consumed separately and alone (Bastian, Collins and Johnson, 2010). Instead, EVOOs are a seasoning and therefore are normally added to food. Moreover, food pairings represent complex mixtures which may also necessitate the exploration of complex dimensions of perception, such as complexity, harmony, or balance (Paulsen et al., 2015). Harmony in food experience has originally been defined as "the pleasant effect made by parts being combined into a whole" (Bullon, 1987). Among experts in the sensory evaluation of food (such as olive oil sensory panellists, sommeliers etc.) harmony is often based on the principle of aromatic similarity, which states that two products with aromatic similarity would be a good match (Eschevins et al., 2018). In olive oil, the concept of harmony has been poorly explored, with a few exceptions (Cerretani et al., 2007; Spinelli, 2014). These recent studies highlight the complexity of dealing with EVOO combinations. However, achieving information on harmonic gastronomic preparations is essential to understand the behavior of raw and cooked ingredients when combined and, therefore, to set up efficient marketing strategies for consumers. Based on the need to further explore the effect of EVOO combinations and the complexity of the food pairing issue, the present work aimed to I. Explore the effect of the combination of EVOOs with raw vegetables on the harmony of the pairings; II. To provide related general indications for harmonic combinations when using EVOO and raw vegetables.

The possibility of providing indications on how to achieve harmonic oil-vegetable pairings may be of interest for anyone who needs to valorize the property of oil at its best, such as field operators and experts (for example chefs, olive oil producers, producers of vegetables preserved in oil) or consumers who use oil as seasoning.

\section{MATERIALS AND METHODS}

\subsection{Assessors and evaluation procedure}

A descriptive analysis was carried out by the trained panel of the University of the Studies "G. D'Annunzio" Chieti-Pescara (Italy), with extensive experience in virgin olive oil tasting (Procida et al., 2016; Procida et al., 2005), according to a procedure by Cerretani et al., (2007). Briefly, assessors evaluated 5 pure EVOOs, 5 vegetables and the same oils combined with the 5 vegetables. EVOOs, vegetables and combinations were evaluated in separate sessions. The assessors joined a total of 26 sessions, including training $(\mathrm{T})$ and individual evaluation sessions (E) specific for each product category: 6 for EVOOs (4 T and $2 \mathrm{E}$ ), 6 for vegetables ( $4 \mathrm{~T}$ and $2 \mathrm{E}$ ), and 14 for oil-vegetable combinations (4 $\mathrm{T}$ and $10 \mathrm{E})$. For each type of product, the training consisted of 4 collective sessions: the first session was used for the vocabulary development, the second session to provide standards for the chosen attributes; the third and fourth sessions were used to calibrate the panel to the scale usage. All samples were evaluated in two replicates, conducted on separate days. Five samples were served within each session. For EVOOs, the panellists evaluated the perceived intensity of 7 descriptors: green fruity odor (o-green odor: defined as the sensations associated with the perception of vegetable notes such as grass, green tomato, tomato leaf, unripe olive), ripe fruity odor (o-ripe: defined as the odor of ripe olive and ripe 
fruits), overall fruity odor (o-overall fruity), bitter taste, sweet taste, pungency, fruity flavor (f-fruity: defined as the overall sensation of fruity perceived after swallowing) on 10-point scales $(1=$ extremely weak, 10 = extremely intense). Odor was defined as the perception of the volatile sensation as orthonasally perceived (o-), while flavor was referred to as the perception of volatile sensations perceived retronasally ( $\mathrm{f}-$ ). When evaluating the fruity sensation of EVOOs, the assessors could freely add the specification for the type of olfactory/flavor sensation.

The attributes for vegetables were sweetness, saltiness, bitterness, sourness, pungency, fatness, flavor intensity, overall persistence. For oil-vegetable combinations, the panellists rated the intensity of the seven descriptors used for EVOOs. After having provided intensity ratings for oil-vegetable combinations, panellists were asked to rate how harmonic/ disharmonic each attribute was perceived to be within the combination using nine-point bipolar scales $(1=$ disharmonic, due to a too low-intensity oil flavor, $5=$ perfectly harmonic, $9=$ disharmonic, due to a high perception of oil flavor). The experiment leader provided the following instruction: "Please, rate how disharmonic/harmonic you perceive each attribute to be within each combination". Values lower than 5 were given by panellists when the flavor of the combination prevailed in intensity over the flavor of the oil; while values higher than 5 were given to pairings when the oil flavor prevailed in intensity over the combination flavor (Cerretani et al., 2007). Evaluations were conducted in individual computerized booths. Samples were served in a randomized and balanced order across judges within each session.

\subsection{Samples}

\subsubsection{Extra virgin olive oils}

Five EVOOs were selected as a function of perceived intensity of bitterness and green flavor based on a previous study (Cerretani et al., 2007). One mild EVOO was inserted twice to check the data repeatability. Four oils were mono-varietal (MV) and one was a commercial blend of Italian oils ("commercial blend"; Bertolli gentile, Carapelli Firenze S.p.A., Firenze, Italy). The mono-varietal EVOOs included the Correggiolo variety ("Correggiolo MV"; Olio Mio 150 Correggiolo, Toscana Enologica Mori, Florence, Italy), the Leccino variety ("Leccino MV"; Olio Mio 150 Leccino, Toscana Enologica Mori, Florence, Italy), Tonda iblea ("Tonda Iblea MV"; Castel di Lego Monti Iblei PDO, Az. Agr. Galioto), the Ghiacciola variety ("Ghiacciola MV"; Nobil Drupa, produced by Consorzio Agrario Brisighellese, Brisighella, Ravenna, Italy). The oils $(30 \mathrm{ml})$ were served in official cups for virgin olive oil tasting (IOC, 2007) at room temperature $\left(22 \pm 1{ }^{\circ} \mathrm{C}\right)$.

\subsubsection{Vegetables}

Five fresh vegetables with increasing intensities of bitter taste were used for the pairing harmony analysis (Table 2). The vegetables included artichokes (median bitter taste $=3$ ), late Treviso radicchio (4), Chioggia radicchio (5), Rocket (Eruca sativa) (6) and early Treviso radicchio (7). Rocket was the only vegetable with a pungent sensation perceived at a high intensity (7). All the vegetables were chopped using a ceramic knife and served raw in sealed transparent cups. For each combination, a weighed amount of vegetables was served $(10 \mathrm{~g})$ per person, and a standardized amount of oil was added to each sample $(7 \mathrm{~g})$, corresponding to a well-dressed portion. Vegetables and oils were gently mixed, and the container was then sealed. Since saltiness showed positive correlations with fruity odor and pungency (Cerretani et al., 2007), combinations were prepared without adding salt, in order to avoid the influence of salt on fruity and pungency. Another reason for avoiding the addition of $\mathrm{NaCl}$ to bitter food is that it inhibits the bitter sensation caused by sodium ions (Lawless and Heymann, 1999). The samples were prepared approximately 30-40 minutes prior to the test. The foods were served in increasing order of bitterness.

\subsection{Score cards}

Specific score cards defined by Cerretani and colleagues (2007) were used for the evaluation of the combinations. The score card was slightly modified by moving the orthonasal olfaction and flavor perceptions before the evaluation of taste and pungency. The score card consisted of category scales with whole numbers to reflect increasing sensory intensity (Lawless and Heymann, 1999). The anchors in the food intensity scales were the result of extensive descriptive panel test sessions. The anchors were meant to avoid the restriction of the panellists' use of the scale, while preserving the highly desirable level of calibration for trained descriptive panellists (Lawless and Heymann, 1999). The anchors also helped in developing a frame of reference for the stimuli and correlated it with the given response (Lawless and Heymann, 1999).

\subsection{Data analysis}

The intensity ratings provided by the trained panel were analyzed by multi-block PCA (Tucker-1) and by $\mathrm{p}^{*} \mathrm{MSE}$ plots (Panel Check software, ver 1.4.0, Nofima, Norway) to assess panel calibration and assessor performance, respectively (Naes, Brockhoff, and Tomic, 2010). Three-way ANOVA models (factors: judge, replicate, product) were applied to verify the absence of a significant effect of replicates and of interactions among factors on the perceived intensities of attributes. After panel data validation, 
the median value was used to describe the perceived intensity of the attributes for EVOOs, vegetables and their combinations. Attribute intensities are expressed as medians in the text because the median is not influenced by extreme values, differently from the mean. Moreover, median values are currently adopted by the official European norms to express the intensity of sensory attributes given by the panel (EC, 2013, Attachment XII). Based on harmony ratings $(\mathrm{H})$ provided by the panellists for each attribute, an Index of Disharmony $\left(I_{d}\right)$ was computed by the experimenter for each pairing (unique for each oil-vegetable combination), which considered the distances of the medians of the 7 descriptors used for the description of oil-vegetable combinations from the central value of 5 (Cerretani et al., 2007). The Index of Disharmony $I_{d}$ was calculated as the sum of the absolute distances of the panel medians for the seven attributes from 5 (perfect harmony):

$$
I_{d}=\frac{\sum_{i=1}^{7}\left|H_{i}-5\right|}{7} \text { where: } I_{d}=\text { Index of }
$$

Disharmony, $\mathrm{H}=$ median harmony score from the panel for each of the seven descriptors within each oil-vegetable combination. While single harmony scores $(\mathrm{H})$ given by panellists ranged from 1 to 9 , the $I_{d}$ was computed once per oil-pairing and it was always a positive value (higher than 0 ) because it represented the sum of absolute distances from perfect harmony for the seven attributes. The higher the $I_{d}$, the higher the distance of the combination from perfect harmony was. A Principal Component Analysis (PCA) was conducted on mean intensity values given to the attributes for the oil-vegetable combinations, inserting the $I_{d}$ (plotted as "Index of Disharmony") as a supplementary variable.

\section{RESULTS}

\subsection{Panel performance}

No case of disagreement among panellists was observed for any of the attributes from the Tucker plot. Considering the $\mathrm{p}^{*} \mathrm{MSE}$ plots, all the assessors were considered reliable. Three-way ANOVA models (factors: judge, replicate, product) separately applied to each food matrix confirmed no significant effect $(p>0.05)$ of replicate or of interaction (Judge*Product, Judge*Replicate, Product*Replicate) on perceived intensities of attributes, and therefore the panel performance was considered valid.

\subsection{Sensory properties of EVOOs}

The median values of the descriptive analysis given by the panel for these EVOOs are shown in Table 1. EVOOs were ranked according to their bitterness intensity as follows: Leccino MV (1), Tonda Iblea MV (2), Correggiolo MV (3), commercial blend (3.5), Ghiacciola MV (4). The Correggiolo MV olive oil showed a green fruity odor with a median value of intensity of 2 and green olfactory perceptions of grass and tomato leaf with an intensity of 1.5; the sweetness median was 1 . Intensities for f-fruity, bitterness, and pungency were centered around 3. The Leccino MV was a very mild EVOO, with a ripe fruity odor of 1 and no green odor/flavor fruity sensations perceivable, with a very low sensation of bitter and pungency (1). The median for sweetness in this sample was 1 .

The Tonda Iblea MV showed the highest intensity for green fruity odor (4) among the oils. Its green fruity flavor was described by the assessors as being characterized by the notes of grass, artichoke, and green tomato with an intensity of approximately 3 . Tonda Iblea MV also had a moderate sweetness (3), bitterness (2) and a moderate-low pungency (1.5).

The Ghiacciola MV had a green fruity odor and flavor of 2, characterized by a typical artichoke odor with an intensity of 2 for taste and mouth feel. Ghacciola MV was characterized by a moderateintense bitterness (4), a moderate pungency (3), a low sweetness (1). The commercial blend had a low green odor (1) and fruity flavor (1), both attributed to the presence of bean pods. The oil was characterized by a moderate bitterness with an intensity of 3.5 , a pungency of 3 , and a sweetness of 3 .

\subsection{Vegetables alone}

The medians of the descriptive analysis given by the panel for the vegetables evaluated alone are shown in Table 2. All vegetables had a certain degree of bitterness (median $\geq 3$ ) characteristic for each matrix. According to the increasing bitterness intensity, the vegetables were ranked as follows: artichoke (3), Late Treviso radicchio (4), Chioggia radicchio (5), Rocket (6), Early Treviso radicchio (7). The variation in intensity of some sensory attributes (sweetness, saltiness, sourness, fatness, overall flavor quality) did not vary greatly among the the selected vegetables (difference in median $\leq 2$ points on discrete scales). Instead, the sensations of bitterness, pungency and overall persistence showed a great variability among the vegetables. Rocket was the most pungent product, described as very pungent (7), while the degree of pungency in Late Treviso radicchio was extremely low (1). The overall flavor persistence was highest in artichoke (8) and lowest in Late Treviso radicchio (3).

\subsection{Food pairings}

When combining EVOOs with the 5 vegetable matrices the following results were found. The $I_{d}$ values for each food and EVOO pairing are shown in Figure 1. The mild EVOO (Leccino MV), with low bitterness and no perceivable green perception, 
TABLE 1. Median values and standard errors of medians for extra virgin olive oils (EVOOs) evaluated alone by a trained panel (eight assessors, two replicates of evaluation). "MV" indicates mono-varietal oils.

\begin{tabular}{|c|c|c|c|c|c|c|c|c|c|c|c|c|c|c|c|c|}
\hline \multirow{4}{*}{$\begin{array}{l}\begin{array}{l}\text { Sensory } \\
\text { qualities }\end{array} \\
\begin{array}{l}\text { Orthonasal } \\
\text { odor }\end{array}\end{array}$} & \multirow{3}{*}{$\frac{\text { Descriptor }^{\mathrm{a}}}{\text { o-green fruity }}$} & \multicolumn{15}{|c|}{ EVOOs } \\
\hline & & \multicolumn{3}{|c|}{ Commercial blend } & \multicolumn{3}{|c|}{ Correggiolo MV } & \multicolumn{3}{|c|}{ Tonda Iblea MV } & \multicolumn{3}{|c|}{ Leccino MV } & \multicolumn{3}{|c|}{ Ghiacciola MV } \\
\hline & & 1.0 & \pm & 0.2 & 2.0 & \pm & 0.3 & 4.0 & \pm & 0.5 & 0.0 & \pm & 0.2 & 2.0 & \pm & 0.1 \\
\hline & o-ripe fruity & 0.0 & \pm & 0.1 & 0.0 & \pm & 0.1 & 0.0 & \pm & 0.2 & 1.0 & \pm & 0.2 & 0.0 & \pm & 0.1 \\
\hline & o-overall fruity & 2.0 & \pm & 0.3 & 2.0 & \pm & 0.4 & 4.0 & \pm & 0.5 & 1.0 & \pm & 0.3 & 2.0 & \pm & 0.3 \\
\hline \multirow[t]{2}{*}{ Taste } & bitterness & 3.5 & \pm & 0.6 & 3.0 & \pm & 0.5 & 2.0 & \pm & 0.3 & 1.0 & \pm & 0.3 & 4.0 & \pm & 0.6 \\
\hline & sweetness & 3.0 & \pm & 0.4 & 1.0 & \pm & 0.1 & 3.0 & \pm & 0.5 & 3.0 & \pm & 0.6 & 1.0 & \pm & 0.3 \\
\hline Mouthfeel & pungency & 3.0 & \pm & 0.5 & 3.0 & \pm & 0.3 & 1.5 & \pm & 0.3 & 1.0 & \pm & 0.2 & 3.0 & \pm & 0.5 \\
\hline Retronasal & f-fruity & 1.0 & \pm & 0.1 & 3.0 & \pm & 0.4 & 3.0 & \pm & 0.4 & 0.0 & \pm & 0.1 & 2.0 & \pm & 0.2 \\
\hline
\end{tabular}

odor

a "o-" means odour; "f-" means flavor.

TABLE 2. Median values and standard errors of medians for various vegetables evaluated alone by a trained panel (eight assessors, two replicates of evaluation).

\begin{tabular}{|c|c|c|c|c|c|c|c|c|c|c|c|c|c|c|c|c|}
\hline \multirow{3}{*}{$\begin{array}{l}\text { Sensory } \\
\text { qualities }\end{array}$} & \multirow{3}{*}{$\begin{array}{l}\text { Descriptor } \\
\text { sweetness }\end{array}$} & \multicolumn{15}{|c|}{ Vegetables } \\
\hline & & \multicolumn{3}{|c|}{ Artichoke } & \multicolumn{3}{|c|}{$\begin{array}{c}\text { Late Treviso } \\
\text { Radicchio }\end{array}$} & \multicolumn{3}{|c|}{$\begin{array}{l}\text { Chioggia } \\
\text { Radicchio }\end{array}$} & \multicolumn{3}{|c|}{ Rocket } & \multicolumn{3}{|c|}{$\begin{array}{c}\text { Early Treviso } \\
\text { Radicchio }\end{array}$} \\
\hline & & 3.0 & \pm & 0.3 & 4.0 & \pm & 0.3 & 3.5 & \pm & 0.1 & 4.0 & \pm & 0.3 & 2.0 & \pm & 0.3 \\
\hline & saltiness & 3.0 & \pm & 0.4 & 2.0 & \pm & 0.1 & 3.0 & \pm & 0.2 & 4.0 & \pm & 0.4 & 3.0 & \pm & 0.4 \\
\hline & bitterness & 3.0 & \pm & 0.2 & 4.0 & \pm & 0.5 & 5.0 & \pm & 0.6 & 6.0 & \pm & 0.5 & 7.0 & \pm & 0.9 \\
\hline & sourness & 5.0 & \pm & 0.5 & 3.0 & \pm & 0.4 & 4.0 & \pm & 0.5 & 4.0 & \pm & 0.3 & 4.0 & \pm & 0.3 \\
\hline \multirow[t]{2}{*}{ Mouthfeel } & pungency & 4.0 & \pm & 0.3 & 1.0 & \pm & 0.2 & 3.0 & \pm & 0.2 & 7.0 & \pm & 0.8 & 3.0 & \pm & 0.3 \\
\hline & fatness & 4.0 & \pm & 0.5 & 2.0 & \pm & 0.2 & 2.0 & \pm & 0.3 & 2.0 & \pm & 0.1 & 3.0 & \pm & 0.2 \\
\hline \multirow{2}{*}{$\begin{array}{l}\text { Other } \\
\text { descriptors }\end{array}$} & overall flavor intensity & 4.0 & \pm & 0.6 & 2.0 & \pm & 0.4 & 3.0 & \pm & 0.4 & 4.0 & \pm & 0.3 & 4.0 & \pm & 0.3 \\
\hline & overall persistence & 8.0 & \pm & 1.1 & 3.0 & \pm & 0.3 & 4.0 & \pm & 0.3 & 6.0 & \pm & 0.8 & 6.0 & \pm & 0.6 \\
\hline
\end{tabular}

was the least harmonic with every bitter food tested in pairing. In particular, the $I_{d}$ was the highest for disharmony when considering its combination with the most bitter vegetable (Early Treviso radicchio). Conversely, Tonda Iblea MV paired well with most vegetables (artichoke, Late Treviso radicchio, Early Treviso radicchio). The best harmony $\left(I_{d}=0\right)$ was reached when coupling rocket with Ghiacciola MV oil. In this combination, both ingredients (oil and vegetable) were characterized by high intensities of bitterness (4 and 6, respectively). Artichoke and Late Treviso radicchio showed similar trends when combined with EVOOs.

Interestingly, the two EVOOs characterized by artichoke olfactory perceptions (Tonda Iblea MV and Ghiacciola MV) showed a better harmony than the EVOO lacking that characteristic when combined with the most bitter vegetables (rocket, Early Treviso radicchio).

The rocket pairing was very interesting, as in the present case it was a food with a perceivable pungency (intensity 7 out of 10). The pairing harmony of rocket was also linked to both the bitterness of the oil and the intensity of the green fruity odor/ flavor (both positively correlated), with Ghiacciola MV and Tonda Iblea MV perceived as most harmonic. Interestingly, the most pungent combination was achieved when combining rocket with Tonda Iblea MV (Rocket_Tonda Iblea MV=6), with Tonda Iblea MV having a low pungency when evaluated as a pure sample.

Unlike Early Treviso radicchio, rocket has a significantly higher pungency which is probably better matched with the pungency of Ghiacciola MV. In the pairing with the Early Treviso Radicchio, the harmony once again followed the green flavor of the EVOO.

PCA showed a high value of total explained variance $(84.8 \%)$, contributed from the first two components, respectively: $73.4 \%$ (PC1) and 11.4\% (PC2). The Bi-plot (Figure 2) showed that most volatile attributes (o-green, o-overall fruity, f-fruity), taste (bitter) and mouth feel sensation (pungency) were positively associated with PC1 and highly correlated to each other. Sweetness was negatively correlated with $\mathrm{PC1}$, while odor of ripeness weighed positively on PC2. These last two attributes were positively correlated with each other. The Index 


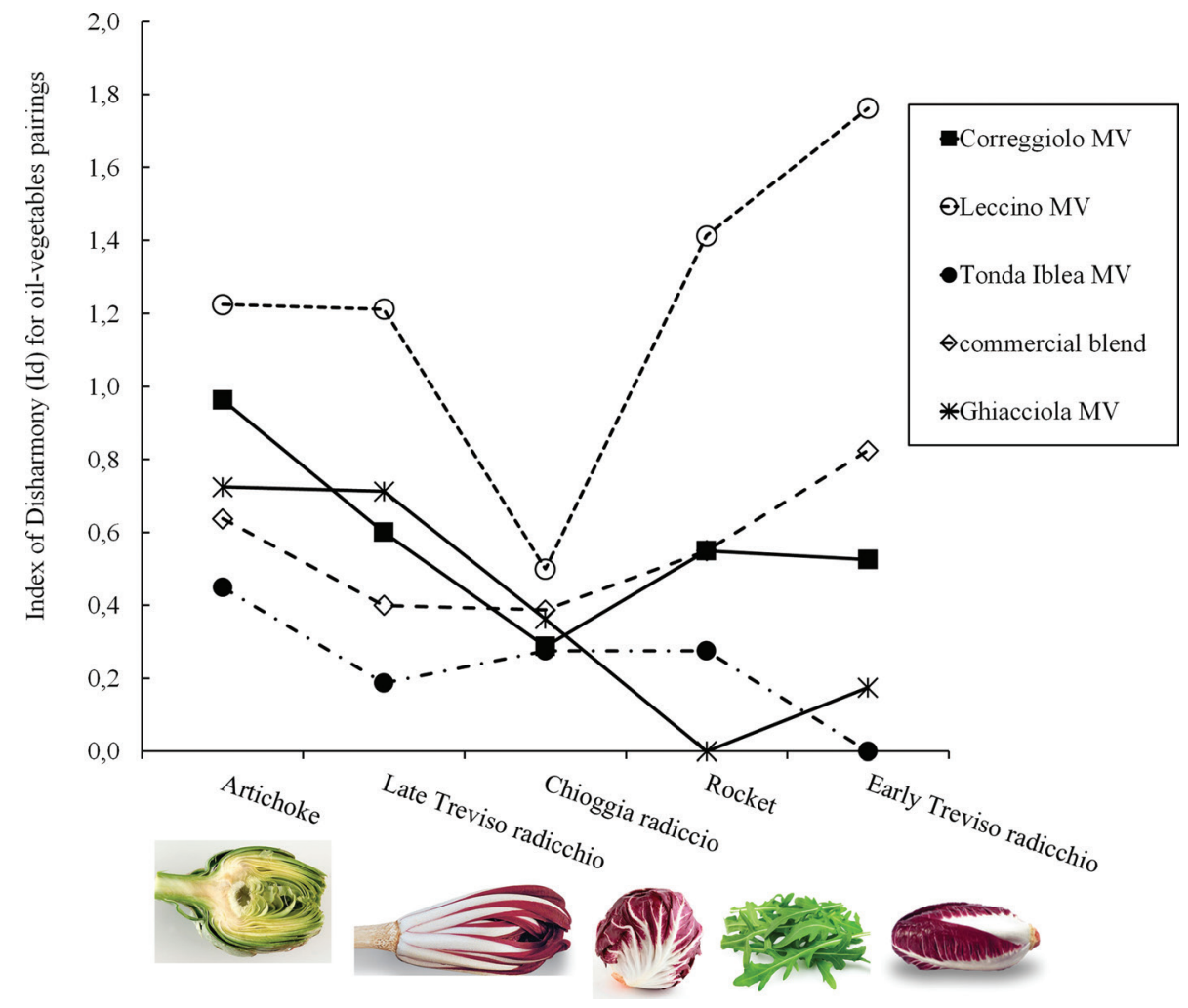

FIGURE 1. Graph for the Index of Disharmony $\left(I_{d}\right)$ of oil-vegetable pairings. Each point depicts the $I_{d}$ value for each corresponding oil-vegetable pairing. $I_{d}$ was computed for each pairing as the sum of the absolute distances of the panel harmony median ratings from 5 (perfect harmony) for the seven considered attributes. $I_{d}$ was based on the evaluation of eight trained assessors on two replicates of evaluation.

of Disharmony value (plotted on the figure as $I_{d}$ ) confirmed that combinations with a low $I_{d}(<0.5$; Early_Tonda Iblea MV, Rocket_Ghiacciola MV, Early Treviso radicchio_Ghiacciola, Late Treviso radicchio_Tonda Iblea MV, Chioggia radicchio_ Correggiolo MV, Chioggia radicchio_Ghiacciola MV, Chioggia radicchio_commercial blend, Late Treviso radicchio_commercial blend, Artichoke Tonda Iblea MV) tended to be positioned on the upper right quadrant of the bi-plot. Therefore, these harmonic pairings were positively and strongly associated to the overall odor perception, to the green fruity odor, to bitterness and to pungency. Instead, most disharmonic pairings $\left(I_{d}>1.0\right.$; Early Treviso radicchio_Leccino MV, Rocket_Leccino MV, Artichoke_Leccino MV, Late Treviso radicchio_Leccino $\mathrm{MV}$ ) were positively associated with the sweet perception and, to a lesser degree, to the odor of ripe fruitiness.

\section{DISCUSSION}

The study provides one of the rare original models for describing the effects of the addition of EVOOs with different sensory characteristics on vegetables and how this addition affects the harmony of the related pairings. EVOOs evaluated alone were characterized by different sensory properties. Leccino MV was the least bitter with a medium-low intensity for fruity and pungency and principally sweet, congruent with a study characterizing Leccino as an olive variety with a low phenolic composition and a medium-high presence of tocopherols (Ranalli et al., 2004). The oil produced from the Correggiolo mono-cultivar has been characterized by a medium-high content of phenolics and tocopherols (Rotondi and Bertazza, 2001). The oil produced from this cultivar is generally characterized by a well-balanced sensory profile between sweetness and pungency, together with a flavor of grass and almond. In accordance with previous studies (Cerretani et al., 2004), the sensory profile of Ghiacciola oil was characterized by very high intensities of olive fruity and bitterness, with a clear pungency, and characteristic green flavors such as grass, artichoke, and green tomatoes. Therefore, the sensory properties of the EVOOs used in the present study confirmed the characterizations found in the literature.

Raw vegetables evaluated alone showed different degrees of bitterness. This was due to the presence of different bitter compounds characteristic of 


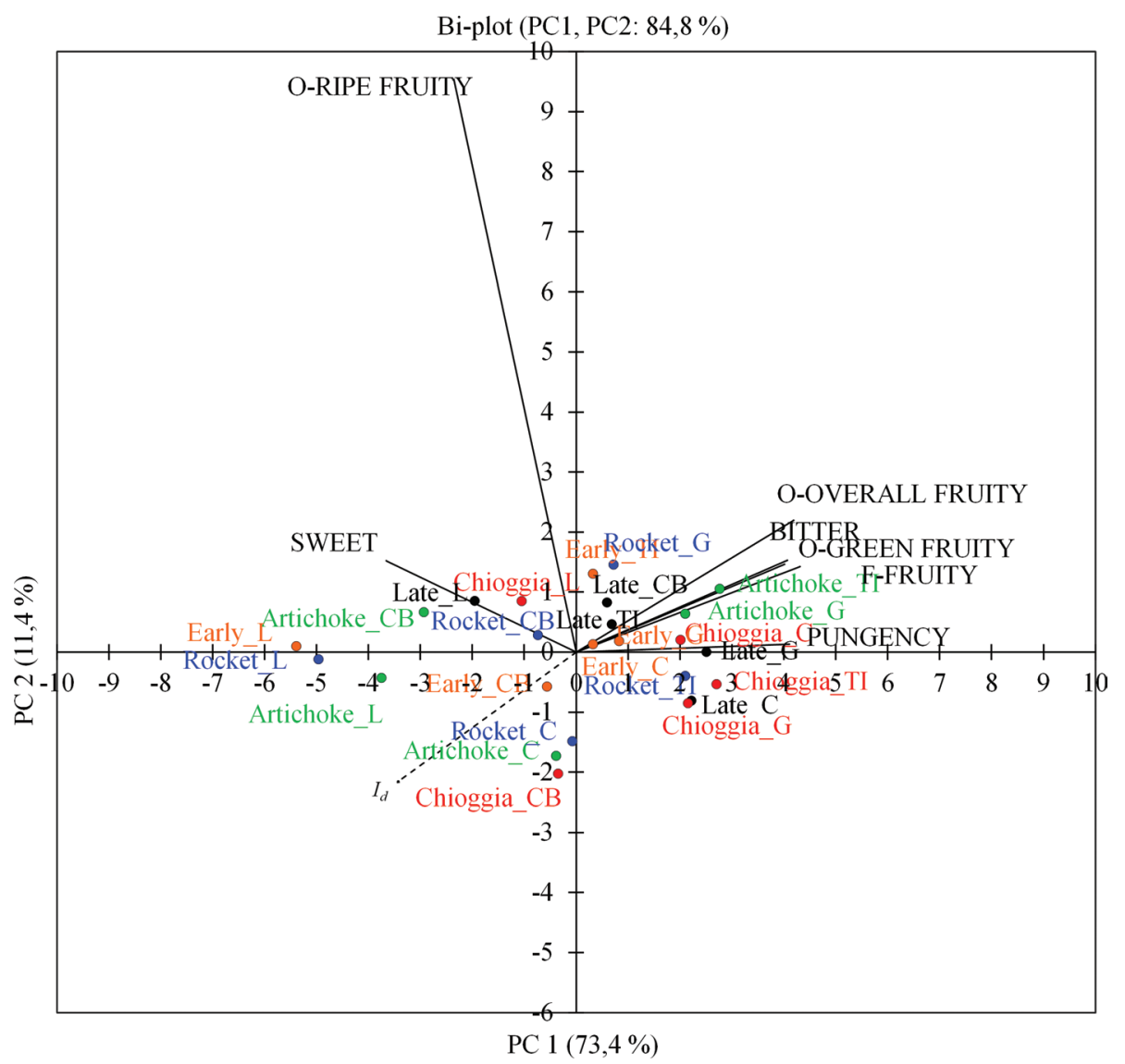

FIgURE 2. Bi-plot obtained from Principal Component Analysis (PCA) of 25 oil-vegetable pairings. Colored points indicate the mean the scores obtained from intensity ratings given by eight trained assessors on two replicates conducted during Descriptive

Analysis. The mean of the evaluation for the Index of Disharmony $\left(I_{d}\right)$ was also inserted as a supplementary variable (dotted line). $I_{d}$ was computed for each pairing as the sum of the absolute distances of the panel harmony median ratings from 5 (perfect harmony) for the seven considered attributes. Names of oil-vegetable pairings show the vegetable followed by the name of oil it is combined with: $\mathrm{C}=$ Correggiolo MV; G=Ghiacciola MV; L=Leccino MV; TI=Tonda Iblea MV; CB=Commercial Blend. Legend: 'o-': orthonasal sensation, 'f-': retronasal sensation.

these matrices, such as glucosinolates (Pasini et al., 2011), isothiocyanates and sulphur compounds in rocket (Bell et al., 2017); sesquiterpene lactones and grosheimin and its derivatives in artichoke (Cravotto et al., 2005) or phenols in radicchio species (Cefola et al., 2016). The choice of the five vegetables was justified as follows: 1. the combination of raw vegetable and raw EVOOs is very common in Italy and part of a normal diet; 2 . some of the chosen vegetables are specific to restricted Italian geographic areas (such as the radicchio species), and therefore their valorization is important for biodiversity.

Indeed, both vegetables and oils can greatly vary in their sensory properties according to different factors (variety, seasonality, geographical area, pedoclimatic factors, growing technique, etc.). However, the raw vegetables and the EVOOs chosen in the present study were extremely diverse, especially for the key attributes of bitterness and green fruity flavor. Thus, both types of products reasonably covered a wide extent of the sensory variability for this type of products.

Moreover, since the official European method for the evaluation of EVOOs (EC, 2013, Attachment XII) is based on the evaluation of fruity, bitter and pungency for the optional classification of an EVOO into "intense", "medium", and "light", EVOOs officially classified according to their flavor can be a priori for the appropriate matrix in order to maximise the harmony of pairing.

It was therefore concluded that the general findings on harmonic pairings are valid regardless of their variability; and that the present approach may represent a possible model to estimate the final harmony of an EVOO-vegetable combination which is applicable on a large-scale based on the currently existing classification of EVOOs.

The combination of oil with vegetables implies complex mechanisms. Interactions between different sensory modalities (smell-smell whether orthonasal or retronasal, smell orthonasal/retronasal-taste, 
chemosensation-taste, etc.) occur during food tasting. Therefore, due to complex physical and perceptual phenomena, the combination of two ingredients can strongly modify the final perception of the combinations in a way which is hardly predictable $a$-priori.

The first study introducing the concept of "sensory functionality" (defined as "the ability to impact on the sensory profile and on the acceptability of the food matrix which it is combined to") was the one conducted by Dinnella and colleagues, and it demonstrated how the addition of EVOOs with different sensory properties strongly modified the sensory properties of the combinations and, consequently, consumer response (Dinnella et al., 2012).

Therefore, since in the combination of EVOOs with other ingredients, unexpected sensory effects can occur, the perception of harmony can be affected by EVOO combinations.

Several definitions of harmony are available in the literature. Lawless introduced the concept of "harmony with contrast" in food experiences (Lawless 2000), described as the desire of experiencing a certain degree of sensory contrasts beyond the sensory balance. This desire originates from typical human nature, characterized contemporarily by a certain degree of neophobia (fear of what is new/ different) and neophilia (interest in what is new). The novelty of the present study relies on the fact that it is one of the very few to provide systematic evidence for the sensory effects which occur when two ingredients coming from two different technological supply chains (horticulture, oliviculture) are combined, which goes beyond the empirical experience of field operators and experts. Moreover, the approach (which requires the computation of an Index of Disharmony) could be expandable to other categories of matrices by focusing on the key attributes characterizing the two food matrices; therefore, the model could be applied to other seasoning-food combinations.

Spinelli has recently reviewed the concept of harmony in culinary uses of EVOOs (Spinelli, 2014), stating that "harmony in food experience is a pleasant effect made by ingredients and products combined into a whole, giving rise to controlled sensory contrasts". Moreover, Spinelli attempted to map the main ways in which EVOOs contribute to increased harmony in gastronomic preparation, grouping mechanisms in four ways: reinforcing an existing sensation, suppressing an existing sensation, enhancing a sensation which is not typical of the oil but of the matrix by contrast, and counter balancing a sensation which is typical of the matrix (Spinelli, 2014). In the present study, the combinations considered envisage the combinations of two ingredients that both had a bitter taste, and the most frequent effect observed tended to be related to the first case (harmonic effect through reinforcement).
In previous work carried out by the authors (Cerretani et al., 2007), the harmony of EVOO coupled with different foods was systematically studied. Based on significant correlations found between specific attributes (bitter taste, green fruitiness) perceived in food and in EVOOs and the perceived harmony of combinations, both bitterness and green fruitiness seemed crucial for harmony in oil-food pairings. Therefore, in the present study, the impact of bitterness and green fruitiness of oils was specifically studied when combined with raw vegetables.

As expected, the mildest EVOO sample (Leccino MV), with the lowest bitterness and no perceivable green perception, was the least harmonic with every bitter food tested in pairings. This confirms the findings of Cerretani et al., (2007) that a bitter food requires a bitter and green EVOO for harmonic pairing.

Previous studies and the taste-smell interaction between bitterness and cut grass odor (cis-3-hexen1-ol) in EVOO suggested that the presence of the cut grass odor (therefore a "green" flavor) caused a significant increase in the perception of bitterness (Caporale, Policastro and Monteleone, 2004). Therefore, in the clear harmonic combination of the bitter vegetable rocket with the oil rich in the bitter and green fruity flavors (Ghiacciola MV) taste-smell interactions may have taken place which produced a highly harmonic combination.

The harmony of green and bitter EVOO with bitter food, identified by Cerretani et al., (2007), has been confirmed in the present work. In particular, when paired with a bitter food, a bitter and green flavor of EVOO increased the overall perception of harmony. When the bitter taste in food was low (below 5), the green fruity flavor of the EVOO was the major factor determining the harmony of the pairing. With food matrices having greater bitter tastes (higher than 5), both bitterness and green fruity flavor of the EVOOs contributed to pairing harmony.

In addition, the best harmony with pungent food was achieved when using oils that have some pungency. Pungency is a chemestetic sensation, originated by the stimulation of the trigeminal nerve (Gerhold and Bautista, 2009), which contributes to increased complexity in model food matrices (Kostyra et al., 2010). Interestingly, in the present study, the most pungent vegetable (rocket) yielded the most pungent pairing, not when combined with the most pungent EVOOs (commercial blend, Correggiolo MV, Ghiacciola MV) but, instead, when combined with Tonda Iblea MV, which had a very low pungency score but showed the highest green fruity flavor. This demonstrated that the volatile sensation of green fruity flavor can significantly enhance the perception of pungency. This finding is similar to a study showing that pungency from capsaicin/chili led to enhancing flavor attributes differently by considering different food matrices (Kostyra et al., 2010). 
In the present study, aromatic similarity (congruency of the same flavor) positively impacted harmony in artichoke, since the best combined oil was the one characterized by a typical artichoke flavor (Tonda Iblea MV). Moreover, very bitter vegetables achieve a high harmony score when combined with a very bitter EVOO or a bitter EVOO with a high green fruity flavor. These findings agreed with a recent study on the combination of food and beverage, demonstrating that the pair sharing an aroma was preferred over the pair with different aromas, and aromatic similarity also increased the pairing's perceived harmony (Eschevins et al., 2018).

The study grouped current approaches for foodbeverages pairing into two types: the first is based on the fact that two products match whenever one product of the pair preserves, or even enhances, the properties of the other; the second refers to the idea that the flavor of the two products should somehow blend into a unique perception (Eschevins et al., 2018). Unlike in our case, the products used in the study of Eschevins and colleagues were artificially created (added with a congruent/discongruent aroma) while in the present study natural vegetable matrices were used (Eschevins et al., 2018). However, we still found that the congruency of the same flavor contributed to harmony.

In general, the present findings confirmed that EVOO with peculiar sensory properties modified the perceived intensity of sensations in the combinations and gave rise to the effects of suppression or contribution to new sensations.

\section{CONCLUSIONS}

The sensory functionality of EVOOs was assessed in combination with raw vegetables. Three main general findings were highlighted, which have a practical implication for everyone interested in achieving harmony when using EVOOs with vegetables (for example chefs, olive oil producers, consumers). The first general finding is that most vegetables achieved the highest harmony scores when coupled with EVOOs according to a "flavor congruency" criterion. This implies that EVOOs characterized by intense green flavor and bitter taste seemed best suited to maximize pairing harmony with very bitter or very pungent vegetables. Moreover, EVOOs characterized by moderate intensity of green flavor perception and bitterness seemed best paired with vegetables characteriszd by low/intermediate bitterness. For future development, it would be interesting to compare consumer perception of harmony of oil-vegetable pairings with harmony evaluated by a trained panel. Due to the complexity in the chemical-physical phenomena occurring when combining different food matrices, the systematic study of the effects of food combinations could help in clarifying frequent phenomena occurring in gastronomy when combining food. Efficient strategies for EVOO valorization should consider these effects of combination for gastronomical purposes.

\section{REFERENCES}

Bastian SEP, Collins C, Johnson TE. 2010. Understanding consumer preferences for Shiraz wine and Cheddar cheese pairings. Food Qual. Pref. 21 (7), 668-678. https://doi. org/10.1016/j.foodqual.2010.02.002

Bell L, Methven L, Signore A, Oruna-Concha MJ, Wagstaff C. 2017. Analysis of seven salad rocket (Eruca sativa) accessions: The relationships between sensory attributes and volatile and non-volatile compounds. Food Chem. 218, 181-191. https://doi.org/10.1016/j.foodchem.2016.09.076

Bullon S. 1978. Longman Dictionary of the Contemporary English, Longman, Harlow, UK.

Caporale G, Policastro S, Monteleone E. 2004. Bitterness enhancement induced by cut grass odorant (cis-3-hexen1-ol) in a model olive oil. Food Qual. Pref. 15 (3), 219-227. https://doi.org/10.1016/S0950-3293(03)00061-2

Cefola M, Carbone V, Minasi P, Pace B. 2016. Phenolic profiles and postharvest quality changes of fresh-cut radicchio (Cichorium intybus L.): nutrient value in fresh vs. stored leaves. J. Food Compos. Anal. 51, 76-84. https://doi. org/10.1016/j.jfca.2016.06.004

Cerretani L, Bendini A, Rotondi A, Mari M, Lercker G, Gallina Toschi T. 2004. Evaluation of the oxidative stability and organoleptic properties of extra-virgin olive oils in relation to olive ripening degree. Prog. Nutr. 6, 50-56. https://doi. org/10.1021/jf0515680

Cerretani L, Biasini G, Bonoli-Carbognin M, Bendini A. 2007. Harmony of virgin olive oil and food pairing: A methodological proposal. J. Sens. Stud. 22 (4), 403-416. https:// doi.org/10.1111/j.1745-459X.2007.00115.x

Commission Regulation (EEC) No 2568/91 of 11 July 1991 on the characteristics of olive oil and olive-residue oil and on the relevant methods of analysis. Official Journal of the European Union, L 248 5.9.1991

Cravotto G, Nano GM, Binello A, Spagliardi P, Seu G. 2005. Chemical and biological modification of cynaropicrin and grosheimin: A structure-bitterness relationship study. J. Sci. Food Agr. 85 (10), 1757-1764. https://doi.org/10.1002/ jsfa. 2180

Dinnella C, Masi C, Zoboli G, Monteleone E. 2012. Sensory functionality of extra-virgin olive oil in vegetable foods assessed by Temporal Dominance of Sensations and Descriptive Analysis. Food Qual. Pref. 26 (2), 141-150. https://doi.org/10.1016/j.foodqual.2012.04.013

Drewnowski A, Gomez-Carneros C. 2000. Bitter taste, phyonutrients, and the consumer: a review. Am.J. Clin. Nutr. 72 (22), 1424-1435. https://doi.org/10.1093/ajcn/72.6.1424

Eschevins A, Giboreau A, Allard T, Dacremont C. 2018. The role of aromatic similarity in food and beverage pairing. Food Qual. Pref. 65, 18-27. https://doi.org/10.1016/j. foodqual.2017.12.005

European Commission, 2013. COMMISSION IMPLEMENTING REGULATION (EU) No 1348/2013 of 16 December 2013 amending Regulation (EEC) No $2568 / 91$ on the characteristics of olive oil and olive-residue oil and on the relevant methods of analysis. Official Journal of the European Union, L 338, 17 December 2013.

Gerhold KA, Bautista DM. 2009. Molecular and cellular mechanisms of trigeminal chemosensation. Ann. NN. Acad. Sci. 1170, 184-189. https://doi.org/10.1111/j.1749-6632.2009. 03895. $\mathrm{x}$

Gutiérrez-Rosales F, Ríos JJ, Gómez-Rey ML. 2003. Main polyphenols in the bitter taste of virgin olive oil. Structural confirmation by on-line high-performance liquid chromatography electrospray ionization mass spectrometry. J. Agric. Food Chem. 51 (20), 6021-6025. https://doi.org/ $10.1021 /$ if021199x

International Olive Council (IOC). 2007. Glass for oil tasting. COI/T.20/DOC. 5/REV.1 - 2007. 
Keller KL, Steinmann L, Nurse RJ, Tepper BJ. 2002. Genetic taste sensitivity to 6-n-propylthiouracil influences food preference and reported intake in preschool children. Appetite 38 (1), 3-12. https://doi.org/10.1006/appe.2001.0441

Kostyra E, Baryłko-Pikielna N, Dabrowska U. 2010. Relationship of pungency and leading flavour attributes in model food matrices - temporal aspects. Food Qual. Pref. 21 (2), 197-206. https://doi.org/10.1016/j.foodqual.2009.03.007

Lawless HT 2000. Sensory combinations in the meal, in: Meiselman, H.D. (Ed.), Dimensions of the Meal: the Science, Culture, Business and Art of Eating, Aspen Publishers, Gaithersburg, MD, 92-106.

Lawless HT, Heymann H. 1999. Sensory evaluation of food: principles and practice. Springer, New York. https://doi. org/10.1007/978-1-4419-6488-5

Naes T, Brockhoff B, Tomic O. 2010. Quality control of sensory profile data. In: Statistics for sensory and consumer science. J. Wiley \& Sons, Chichester, UK, pp.11-38.

Pasini F, Verardo V, Cerretani L, Caboni MF, D'Antuono LF. 2011. Rocket salad (Diplotaxis and Eruca spp.) sensory analysis and relation with glucosinolate and phenolic content. J. Sci. Food Agr. 91 (15), 2858-2864. https://doi.org/ $10.1002 /$ isfa 4535

Paulsen MT, Rognså GH, Hersleth M. 2015. Consumer perception of food-beverage pairings: The influence of unity in variety and balance. Int. J. Gast. Food Sci. 2, 83-92. https:// doi.org/10.1016/j.ijgfs.2014.12.003

Procida G, Giomo A, Cichelli A, Conte LS. 2005. Study of volatile compounds of defective virgin olive oils and sensory evaluation: A chemometric approach. J. Sci. Food Agr. 85 (13), 2175-2183. https://doi.org/10.1002/jsfa.2122
Procida G, Cichelli A, Lagazio C, Conte L. 2016. Relationships between volatile compounds and sensory characteristics in virgin olive oil by analytical and chemometric approaches. J. Sci. Food Agr. 96 (1), 311-318. https://doi. org/10.1002/jsfa.7096

Ranalli A, Lucera L, Contento S, Simone N, Del Re P. 2004. Bioactive constituents, flavors and aromas of virgin oils obtained by processing olives with a natural enzyme extract. Eur. J. Lipid Sci. Technol. 106, 187-197. https://doi. org/10.1002/ejlt.200300863

Recchia A, Monteleone E, Tuorila H. 2012. Responses to extra virgin olive oils in consumers with varying commitment to oils. Food Qual. Pref. 24 (1), 53-161. https://doi. org/10.1016/j.foodqual.2011.10.009

Rotondi A, Bertazza G. 2001. Caratteri chimici dell'olio di oliva. In: La qualità dell'olio extravergine di oliva dei colli riminesi: la cultivar Correggiolo. Cristoferi, G. Ed. La Mandragora, Bologna, Italy, 87-99 [Rotondi A, Bertazza G. 2001. Chemical characteristics of olive oil. In: The quality of extra virgin olive oils from the Rimini hills: the Corregiolo variety. Edited by Cristoferi, G. La Mandragora, Bologna, Italy, pp. 87-99]

Spinelli S. 2014. Investigating the culinary use of olive oils. In: Olive Oil Sensory Science. Eds: Monteleone E, Langstaff S.. Wiley Blackwell, Jhon Wiley \& Sons, Ldt, Chichester, UK, pp. 195-225. https://doi.org/10.1002/9781118332511

Van Duyn MAS, Pivonka E. 2000. Overview of the Health Benefits of Fruit and Vegetable Consumption for the Dietetics Professional Selected Literature. J. Am. Diet. Assoc. 100 (12), 1511-1521. https://doi.org/10.1016/S00028223(00)00420-X 